\title{
Performance Analysis of Space Time Trellis Codes Over Nakagami Fading \& Rayleigh Fading Channels: With Comparative Analysis
}

\author{
Prof. Ashutosh Sharma \\ Department of Electronics, \\ Barkatullah University, \\ Bhopal India
}

\author{
Dr.Poonam Sinha \\ Department of Electronics \\ Barkatullah University, \\ Bhopal India
}

\begin{abstract}
Space-time trellis codes provide both diversity gain and coding gain. There are two different design criteria proposed for spacetime trellis codes (STTCs), namely the rank and determinant criteria (RDC) and the Euclidean distance design criteria (EDC). In this paper, we present the performance of STTCs over Nakagami fading channels. Our results show that the STTCs designed for Rayleigh fading channels \& Recian fading channels are also suitable for Nakagami fading channels. Nakagami fading channel models are considered more versatile than other channel models. In Gong et al. presented the performance of the STTCs over Nakagami fading channels. In this paper, we also present the performance of the STTCs designed using the EDC over Nakagami fading channels.
\end{abstract}

\section{KEYWORDS}

Wireless, Space-time trellis codes, Nakagami fading, space time coding.

\section{INTRODUCTION}

A typical STTC based wireless system has an encoder, pulse shaper, modulator and multiple transmit antennas at the transmitter, and the receiver has one or more receive antennas, demodulator, channel estimator and STTC decoder. We consider a mobile communication system with $n_{t}$ transmit antennas and $n_{r}$ receive antennas as shown in Figures 1 (a) and (b). The space-time trellis encoder encodes the data $s(t)$ coming from the information source and the encoded data is divided into $n_{t}$ streams of data

$$
\begin{array}{lllll}
c_{t}^{1} & c_{t}^{2} & \ldots \ldots & c_{t}^{n_{t}}
\end{array} .
$$

Each of these streams of data passes through a pulse shaper before being modulated. The output of modulator $i$ at time slot $t$ is the signal $c_{t}^{i}$, which transmitted through is transmit antenna $i$. Here $n_{t} 1 \leq i \leq n_{t}$ The transmitted symbols have energy $E_{t}$. We assume that the $n_{t}$ signals are transmitted simultaneously from the antennas.

The signals have transmission period $T$. In the receiver, each antenna receives a superposition of $n_{t}$ transmitted signals corrupted by noise and multipath fading. Let the complex channel coefficient between transmit antenna $i$ and receive antenna $\mathrm{j}$ have a value of $h_{i, j}(t)$ at time $t$, where $1 \leq i \leq n_{r}$. The received signal at antenna $j, j=1,2, \ldots \ldots ., n_{r}$ G.L. Stuber [1] is then

$$
r_{t}=\sqrt{E_{s}} \sum_{i=1}^{n} h_{i, j}(t) c_{t}^{i}(t)+\eta_{t}^{j}
$$

Where $\eta_{t}^{j}$ is additive white Gaussian noise (AWGN) at receive antenna $j$, which has zero mean and power spectral density $N_{0}$ and $\boldsymbol{h}_{i, j}(\boldsymbol{t})$ channel coefficient between transmit and receive antennas time, frequency and spatial diversity are the traditional strategies to combat multipath fading, which is a major obstacle to high data rates over mobile communication channels. Spacetime trellis codes (STTCs) were first proposed in [1] and combine channel coding, modulation, transmit diversity, and optional receive diversity. There has been rapid progress in this field resulting in codes with greater coding gain than those provided in [1], in particular Baro et al. [2]. The STTCs in [1] and [2] were designed using the rank and determinant criteria (RDC). Chen et al. [3] proposed a new design criteria which is known as the Euclidean distance criteria (EDC). Codes designed using the EDC show a significant performance improvement over those based on the RDC.

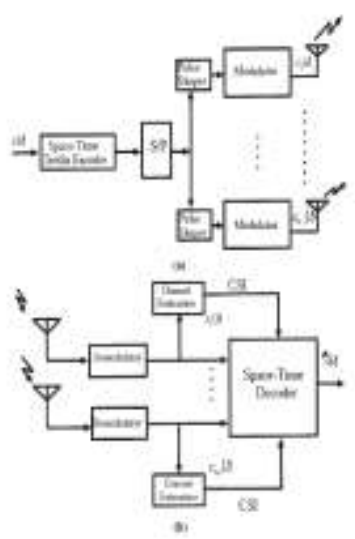

Figure 1 A Block diagram of (a) transmitter (b) Receiver of a STTC based system

(a) Code Construction

STTCs are represented in a number of ways, such as the trellis form or generator matrix form as illustrated in Figure 3.2 for a simple STTC. In N. Seshadri, V. Tarokh, A.R. Calderbank [9], 
most codes are presented in trellis form. But for a systematic code search, the generator matrix form is preferable. The generator matrix representation is also used for convolutional codes. However the generator matrix notation as shown in Figure 2 (a) is a little different than that used for convolutional codes $\mathrm{S}$. Baro, G. Bauch, A. Hansmann [16] [17]. In Figure 2 (a) two input bits enter the encoder every symbol period[6]. The input streams are multiplied by the branch coefficients, which can be put into a matrix form (generator matrix) as shown below:

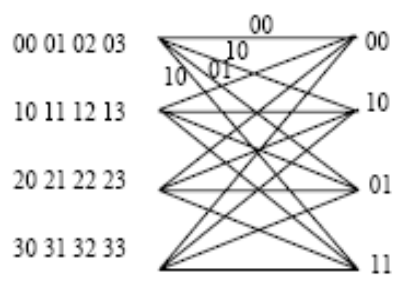

a) Trellis diagram

Fig 2. A Trellis Diagram \& Generator matrix description of simple space time trellis Code

$$
G=\left[\begin{array}{llll}
a_{0}^{1} & b_{0}^{1} & a_{1}^{1} & b_{1}^{1} \\
a_{0}^{2} & b_{0}^{2} & a_{1}^{2} & b_{1}^{2}
\end{array}\right]=\left[\begin{array}{llll}
0 & 0 & 2 & 1 \\
2 & 1 & 0 & 0
\end{array}\right]
$$

\section{(b) Generator Matrix}

The following example illustrates STTC encoding. In Figure 3 we provide a trellis diagram and a table of output symbols related to the input bits and current state. This trellis is for 4PSK constellations. Let the input symbol stream to the encoder is $\left[\begin{array}{lllllll}2 & 3 & 2 & 1 & 0 & 1 & \ldots . . . .\end{array}\right]$. Initially the encoder is in state " 0 ". Thus " 0 " will be transmitted from the first antenna, the second antenna transmits " 2 " and the encoder goes into state " 2 " D. Varshne, C. Arumugam, V. Vijayaraghavan, N. Vijay and S. Srikanth [15]. In this way for this input symbol stream the output for the 4-PSK STTC is as follows

$$
c=\left[\begin{array}{rrrrrr}
0 & 2 & 3 & 1 & 0 & 1 \\
2 & 3 & 1 & 0 & 1 & \ldots
\end{array}\right]
$$

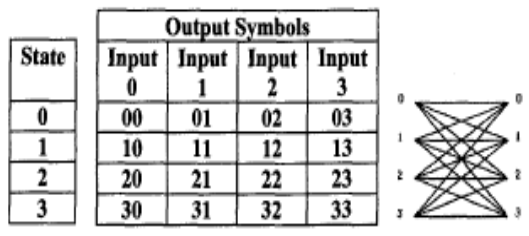

Fig 3. 4-PSK 4-State STTC

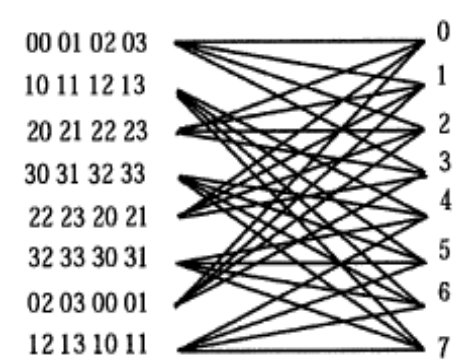

Fig 4. Trellis Diagram of 4-PSK 8 State STTC

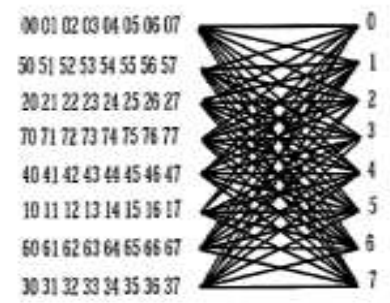

Fig 5 Trellis Diagram of 4-PSK 8 State STTC

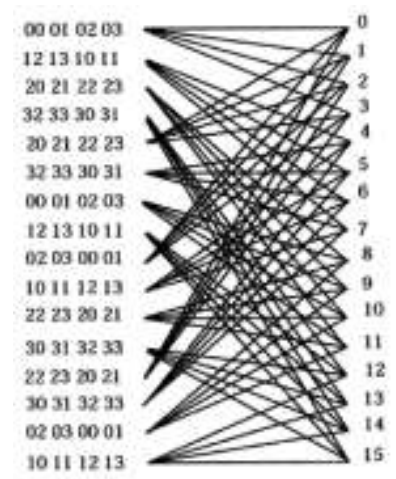

Fig 6 Trellis Diagram of 4-PSK 16 State STTC

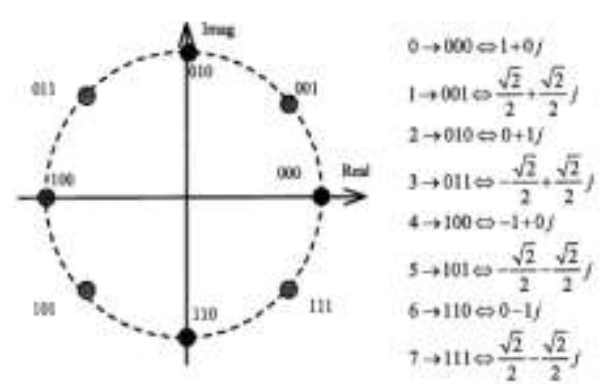

Fig 7. 8 PSK Signal Constellation 


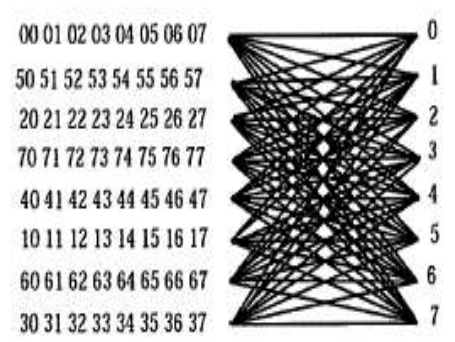

Fig 8 Trellis Diagram of a 8-PSK 8 State STTC

\section{PERFORMANCE CRITERIA}

We assume that the STTC codeword is given by

$$
c=\left(c_{1}^{1} c_{1}^{2} \ldots . c_{1}^{n_{t}} c_{2}^{1} c_{2}^{2} \ldots . . c_{2}^{n_{t}} \ldots \ldots . c_{l}^{1} c_{l}^{2} \ldots . c_{l}^{n_{t}}\right)
$$

Where $l$ is the frame length. We consider a maximum likelihood receiver, which may possibly decide on an erroneous code word $\boldsymbol{e}$ , given by

$$
e=\left(e_{1}^{1} e_{1}^{2} \ldots . e_{1}^{n_{t}} e_{2}^{1} e_{2}^{2} \ldots . . e_{2}^{n_{t}} \ldots \ldots . e_{l}^{1} e_{l}^{2} \ldots . e_{l}^{n_{t}}\right)
$$

We can write the difference code matrix, the difference between the erroneous codeword and the transmitted codeword as follows-

$$
B(c, e)=\left(\begin{array}{cccc}
e_{1}^{1}-c_{1}^{1} & e_{2}^{1}-c_{2}^{1} & \ldots \ldots & e_{l}^{1}-c_{l}^{1} \\
e_{1}^{2}-c_{1}^{2} & e_{2}^{2}-c_{2}^{2} & \ldots \ldots & e_{l}^{2}-c_{l}^{2} \\
e_{1}^{3}-c_{1}^{3} & e_{2}^{3}-c_{2}^{3} & \ldots \ldots & e_{l}^{3}-c_{l}^{3} \\
\cdot & . & \ldots \ldots . & \cdot \\
\cdot & . & \ldots \ldots . & . \\
\cdot & . & \ldots \ldots . & . \\
e_{1}^{n_{t}}-c_{1}^{n_{t}} & e_{2}^{n_{t}}-c_{2}^{n_{t}} & \ldots \ldots . & e_{l}^{n_{t}}-c_{l}^{n_{t}}
\end{array}\right)
$$

The difference matrix $B(c, e)$ has dimension $n_{t} \times l$. From N. Seshadri, V. Tarokh, A.R. Calderbank [9] we know that to achieve the maximum diversity order $n_{r,} n_{t} n_{r}$, receive antennas, $n_{t}$ transmit antennas) matrix $B(c, e)$ must have full rank for all possible codewords $\mathrm{c}$ and $\mathrm{e}$. If $B(c, e)$ has minimum rank $\mathrm{r}$ over the set of pairs of distinct codewords then the diversity will be $r . n_{r}$ N. Seshadri, V. Tarokh, A.R. Calderbank [9] [7].

Let $A(c, e)=B(c, e) B^{*}(c, e)$ be the distance matrix, where $B^{*}(c, e)$ is the Hermitian of $B(c, e)$. The rank of $A(c, e)$ is $r$. A has minimum dimension $n_{t}-r$ and exactly $n_{t}-r$ eigen values of $\mathrm{A}$ are zero. The non-zero eigenvalues of $\mathrm{A}$ are denoted by $\lambda_{1}, \lambda_{2}, \lambda_{3} \ldots \ldots . \lambda_{n}$. Assuming perfect channel state information (CSI), the probability of transmitting $\mathrm{c}$ and deciding on an erroneous codeword e at the decoder is given by V. Tarokh, N. Seshadri, A. R. Calderbank [5], V. Tarokh, N. Seshadri, A. R. Calderbank [9], Z. Chen, J. Yuan, B. Vucetic [19]

$$
\begin{gathered}
P\left(c, e \mid h_{i, j}, i=1,2, \ldots ., n_{t} \text { and } j=1,2, \ldots, n_{r}\right)=Q\left(\sqrt{\frac{E_{s}}{2 N_{0}} d^{2}(c, e)}\right), \\
\text { Or } \\
P\left(c \rightarrow e \mid h_{i, j}, i=1,2, \ldots ., n_{t} \text { and } j=1,2, \ldots ., n_{r}\right) \leq \exp \left(-d^{2}(c, e) E_{s} / 4 N_{0}\right)
\end{gathered}
$$

Where $N_{0} / 2$ is the noise variance per dimension and

$$
d^{2}(c, e)=\sum_{j=1}^{n_{r}} \sum_{t=1}^{l}\left|\sum_{i=1}^{n_{t}} h_{i, j}\left(c_{t}^{i}-e_{t}^{i}\right)\right|^{2}
$$

is the Euclidean distance. For independent Rician fading we can be as

$$
P(c \rightarrow e) \leq \prod_{j=1}^{n_{f}}\left(\prod_{i=1}^{n_{i}} \frac{1}{1+\frac{E_{s}}{4 N_{0}} \lambda_{i}} \exp \left(-\frac{K_{i, j} \frac{E_{s}}{4 N_{0}} \lambda_{i}}{1+\frac{E_{s}}{4 N_{0}} \lambda_{i}}\right)\right)
$$

Here $K_{i, j}$ is a coefficient and it is described in details in V. Tarokh, N. Seshadri, A. R. Calderbank [5] .

For the special case of Rayleigh fading we can assume $K_{i, j}=0$ for all i and j V. Tarokh, N. Seshadri, A. R. Calderbank [5] . Then (8) can be written as

$$
P(c \rightarrow e) \leq\left(\frac{1}{\prod_{i=1}^{n_{t}} 1+\frac{E_{s}}{4 N_{0}} \lambda_{i}}\right)^{n_{r}}
$$

Let $\boldsymbol{r}$ denote the rank of matrix $A(c, e)$. The matrix $A$ has dimension $n_{t}-r$ and $n_{t}-r$ eigen values of $\boldsymbol{A}$ are zero.

$P(c \rightarrow e) \leq\left(\prod_{i=1}^{r} \lambda_{i}\right)^{-n_{r}}\left(\frac{E_{s}}{4 N_{0}}\right)^{-r m_{r}}$

We can derive following Design criteria for the STTC to achieve the best performance of a given system V. Tarokh, N. Seshadri, A. R. Calderbank [5].

\section{A. STTC over Nakagami Fading Channels}

In the multipath fading channel model literature, the Rayleigh and Rician distributions are frequently used. However, the Nakagami model is often more versatile than the above mentioned channels Y. Gong and K. B. Letaief [3]. In this model it is assumed that the received signal is the sum of vectors with 
random amplitudes and random phases[8]. This assumption makes this model more flexible than the Rayleigh and Rician distributions M. Nakagami [3]. The Nakagami distribution is given by

$$
p(\alpha)=\frac{2}{\Gamma(M)}\left(\frac{m}{\Omega}\right)^{m} \alpha^{2 m-1} e^{-\alpha^{2} m / \Omega}
$$

Where $\Gamma(x)$ denotes the Gamma function of $\mathrm{x}$, and

$$
\begin{aligned}
& \Omega=E\left[\alpha^{2}\right], \\
& m=\frac{\Omega^{2}}{E\left[\alpha^{2}-\Omega^{2}\right]}, \quad m \geq 1 / 2
\end{aligned}
$$

The notation $E[x]$ denotes the expected value of $\mathrm{x}$. The constant $m$ is called the inverse fading parameter [10] with $m=1$ and $m=\infty$ corresponding to Rayleigh fading and no fading, respectively.

\section{B. Independent Fading}

The amplitudes of $h_{i, j}$ are identical and independent $\mathrm{m}$ -

distributed with the same $m$ and $\Omega$. Pair wise error probability is given by Y. Gong and K. B. Letaief [3],

$$
P(c \rightarrow e) \leq \frac{m / \Omega}{\Gamma(m)} \prod_{j=1}^{m} \prod_{i=1}^{n} \frac{\Gamma(m)}{\left(\frac{E_{s}}{4 N_{0}} \lambda_{1}+\frac{m}{\Omega}\right)^{m}}
$$

Here $\quad \Gamma(m)$ is gamma function, which means $\Gamma(n)=\int_{0}^{\infty} t^{n-1} e^{-t} d t, n>0$. Equation

$$
\begin{aligned}
P(c \rightarrow e) & \leq f(m)\left(\prod_{i=1}^{r}\left(\lambda_{i}+\frac{4 N_{0} m}{E_{s} \Omega}\right)\right)^{-m n_{r}}\left(\frac{E_{s}}{4 N_{0}}\right)^{-r m n_{r}} \\
& \leq f(m)\left(\prod_{i=1}^{r} \lambda_{i}\right)^{-m n_{r}}\left(\frac{E_{s}}{4 N_{0}}\right)^{-r m n_{r}}
\end{aligned}
$$

Here $f(m)=m / \Omega{ }^{m-m n_{r}\left(n_{t}-r\right)} \Gamma(m)^{n_{t} n_{r}-1} \quad \mathrm{Y}$. Gong and K. B. Letaief [3].

From (12.a), the diversity order is $r m n_{r}$. This is in fact $m$ times the diversity order that can be achieved in Rayleigh fading. The coding gain is $f(m)=m / \Omega^{-1 / r m n_{r}}\left(\prod_{i=1}^{r} \lambda_{i}\right)^{1 / r} \mathrm{~J}$. Yuan, Chen and B. Vucetic [22]. Now $m=1$ and $\Omega=1$, we can simplify (12.b) as Y. Gong and K. B. Letaief [3].

$$
P(c \rightarrow e) \leq\left(\prod_{i=1}^{r} \lambda_{i}\right)^{-n_{r}}\left(\frac{E_{s}}{4 N_{0}}\right)^{-r n_{r}}
$$

This agrees with (13) which is the error probability for Rayleigh fading. If we compare (12.a) and (12.b) we see that the only difference between them is the factor $f(m)$ and $m$ on the right hand side of (2.14). Thus we can say that the diversity order achieved in Rayleigh fading increases by a factor of $\mathrm{m}$ in Nakagami fading and the coding gain is multiplied by a factor of $f(m)^{-1 / r m n_{r}}$. We can consider this factor as the additional coding gain due to Nakagarni fading [3] [11].

\section{Correlated Fading}

In this section we present the design criteria for STTC in correlated fading. We considered the case when the fading coefficients, $h_{i, j}$ are correlated. We assume that the envelopes of $h_{i, j}$ are modeled as identically correlated Nakagami distributed random variables Y. Gong and K. B. Letaief [3]. In Y. Gong and K. B. Letaief [3] it is shown that rank and determinant criteria is similar to the independent Nakagami fading criteria.

\section{STTC DECODER}

The decoder is based on the Viterbi algorithm, so it uses the trellis structure of the code. Each time the decoder receives a pair of channel symbols it computes a metric to measure the "distance" between what is received and all of the possible channel symbol pairs that could have been transmitted[14]. For hard decision Viterbi decoding the Hamming distance is used, and the Euclidean distance is used for soft decision Viterbi decoding. The metric values computed for the paths between the states at the previous time instant and the states at the current time instant are called "branch metrics". We assume that the decoder has ideal channel state information (CSI) and thus knows the path gains $h_{i, j}\left(\right.$ where $i=1,2, \ldots \ldots \ldots, n_{t}$ and $\left.j=1,2, \ldots \ldots, n_{r}\right)$. If the signal is $r_{t}^{j}$ at receive antenna $\mathrm{j}$ and time $\boldsymbol{t}$, the branch metric for a transition labeled $x_{t}^{1} x_{t}^{2} \ldots \ldots . . x_{t}^{n_{t}}$ is given by G.L. Stuber [1].

$$
\sum_{j=1}^{n_{r}}\left|r_{t}^{j}-\sum_{i=1}^{n_{t}} h_{i, j} q_{t}^{i}\right|^{2}
$$

The Viterbi algorithm determines the path with the lowest accumulated metric.

\section{Simulation System Model}

The simulation is carried out in MATLAB. The simulation system model is illustrated in Figure .

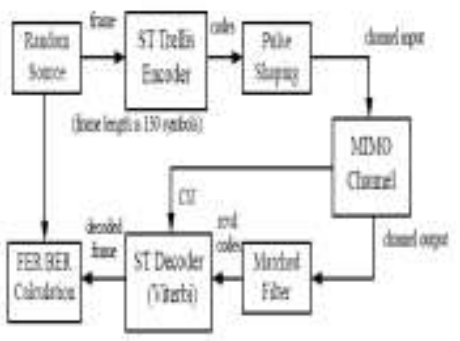

Fig 9.Simulation system model

Random M-PSK symbols are grouped into frames, which consists of 130 symbols each. The space-time encoder takes the 
frame as input and generates codeword pairs for each input symbol simultaneously for all the transmit antennas. Pulse shaping and matched filter are used for simulation over frequency selective fading channels. These complex signals are transmitted through the MIMO channel. The signals and channels are modeled in base-band, thus modulation/demodulation operations are not carried out. Channels used in this project include flat Rayleigh/Nakagami fading channels and two-ray model frequency selective fading channel. [20] [3]

We assume that perfect channel state information (CSI) is available at the receiver. At the receiver, a maximum likelihood sequence detector is used to decode the received signal. A modified vector Viterbi decoder is employed. Error probability calculation is carried out after decoding each frame. [20] [18]

\section{C-MEX function}

MATLAB is a versatile integrated environment for computer simulation and offers rich built-in functions for various applications. Most common procedures in signal processing, encoding/decoding and modulation/demodulation are provided. However, when built-in functions are not appropriate for certain applications and detailed algorithm is written in MATLAB scripts (M-files), which contain a lot of loops, efficiency becomes a critical problem. Even a simple program can take a very long simulation time, thus the accuracy may be easily compromised. [20] [21]

The Viterbi decoding algorithm for space-time coding is computational complex. In order to speed up the simulation, the main encoder/decoder of space-time coding are implemented as $\mathrm{C}$ subroutines and compiled into dynamically linked (DLL) files, which are referred to as MEX functions in MATLAB. In this approach, the encoder/decoder subroutines can be called directly in MATLAB as if they were built-in functions.

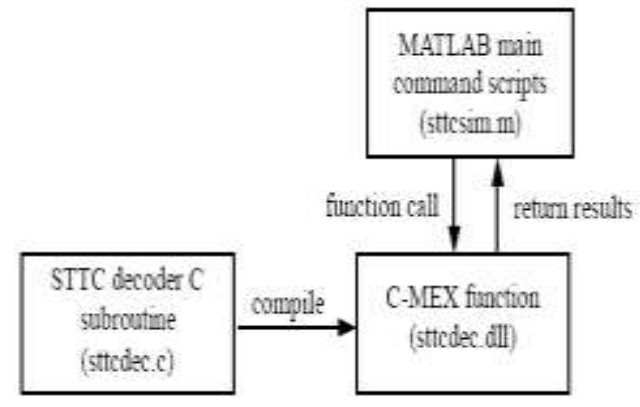

Fig 10 Illustration of C-MEX function in MATLAB

\section{IV.STTC ENCODER}

The STTC encoder uses a trellis structure similar with the trellis structure for encoding/decoding convolution codes used in MATLAB. The STTC encoder is adopted from the convolution encoder in MATLAB .

The encoder assumes that the M-PSK constellation is used for the space-time codes. The encoder takes L M-PSK symbols (a frame) as input, and generates a matrix of $L \times N$ complex codes for $\mathrm{N}$ transmit antenna. The encoding operates on a framebased input and the starting state can be specified. The encoding procedure is described below:

1) Look up the output for each transmit antenna for the current input and state.

2) Look up the next state for the current input and update the state.

3) Loop for next input symbol until the end of frame is reached.

\section{V.STTC DECODER}

The STTC decoder also uses the trellis structure for the Viterbi algorithm. The STTC decoder is adopted from the Viterbi decoder for convolution codes in MATLAB.

The description of Viterbi algorithm can be found in Seshadri N, Tarokh V, Calderbank AR. [1]. The Viterbi decoder for spacetime coding is different from the conventional convolution decoder in that the branch metric is compute from vector complex inputs and channel state information. The branch metric for $\mathrm{r}$-th receive antenna is:

$$
m(r)=\sum_{j=1}^{M}\left|y_{j r}-\sum_{i=1}^{N} h_{i, j} x_{i r}\right|^{2}
$$

in which $y_{i r}$ is the received signal, $x_{i r}$ is the transmitted signal, $h_{i, j}$ is the channel coefficients. Thus we can see that the computation complex for the branch metric is a major factor for difficult implementation of space-time trellis decoders.

\section{SIMULATION PARAMETERS}

In our simulations we considered the IS-136 standard Y. Gong and K. B. Letaief [3]. In this system, performance is measured by the frame error rate (FER) for a frame consisting of 130 symbols. We also assumed ideal channel state information (CSI) is available at the receiver. We carried out the simulation by MATLAB. Random M-PSK symbols are set in frames as a group, which consists of 130 symbols each. The space-time encoder takes the frame as input and generates codeword pairs of each input symbol simultaneously for all the transmit antennas. Pulse shaping and matched filter are used. These complex signals are transmitted through the MIMO channel. We modeled the signals and channels in base-band. So modulation/demodulation operations are not carried out. We used Monte Carlo simulation to carry out the FER evaluation of the space-time coded system. The FER is given by

$$
p_{e}=\lim _{F \rightarrow \infty} \frac{F_{e}}{F}
$$

Where $\mathrm{F}$ is the total number of transmitted frames and $\mathrm{Fe}$ is the total number of erroneous frames received at the receiver. It is 
impossible to run the simulation for an infinite length of time, so we take $\mathrm{F}$ as a very large number. The maximum number of iterations used was 50,000 for a FER above $10^{-3}$.

\section{A.STTC Performance over Nakagami Channels}

The performance over independent and correlated Nakagami fading channels of the 4-state 4-PSK and 8 -state 4 PSK codes is given with two transmit antennas. In this section, we show the performance of the $4 / 8 / 16 / 32$ states codes presented.

The codes of Table 1 were designed using the Rank and Determinant Criteria (RDC) and these outperform the codes in Table 3 which were designed using the Euclidean Distance Criteria (EDC) for system with $n_{r}=1$. According to S. M. Alamouti [12], when $r n_{r}$, is sufficiently large $>3$ performance of STTCs are dominated by the minimum Euclidean distance of $A(c, e)$ taken over all pairs of distinct codewords $\mathrm{c}$ and $\mathrm{e}$. Here we see for $n_{t}=2$ and $n_{r}=1$ the product $r n_{r}$, is not $\operatorname{large}_{\left(\boldsymbol{r}=n_{t}\right)}$. That's why codes from Table 3, which are designed by EDC is not performing worse than codes from Table 1.

For $\mathrm{m}=2$ and $\mathrm{n}_{\mathrm{t}}=2$, the STTC of Table 2 showed a $0.2 \mathrm{~dB}$ gain over the STTCs from Table 1. This shows that the code design criterion presented in Chen, B. Vucetic, J. Yuan and Lo. Ka. Leong [21] for a Rayleigh fading channel is also valid for Nakagami fading channel Y. Gong and K. B. Letaief [3].

\section{VI.SIMULATION RESULTS IN MATLAB}

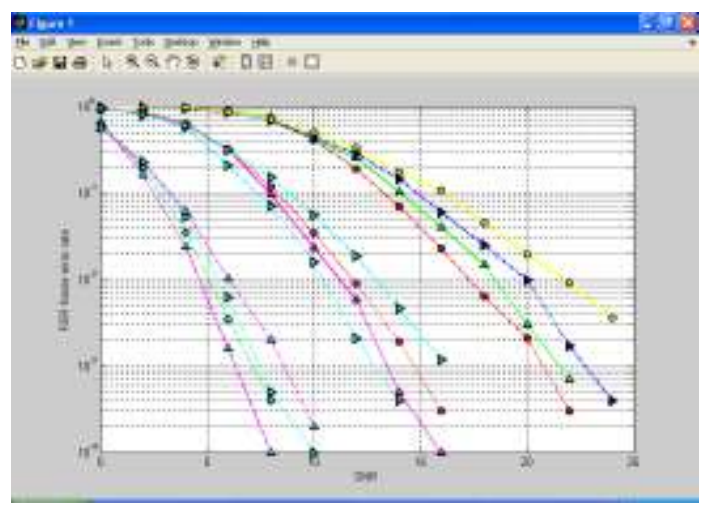

Fig 11 Performance Comparison of the 4-PSK 4-state STTCs from Table 2 (Chen et al.) over correlated Nakagami fading channels $(\mathrm{m}=2)$ for $\rho=0,0.5,0.8$ and 1.0 with

$$
n_{t}=2, n_{r}=1,2 \text { and } 4 \text {. }
$$

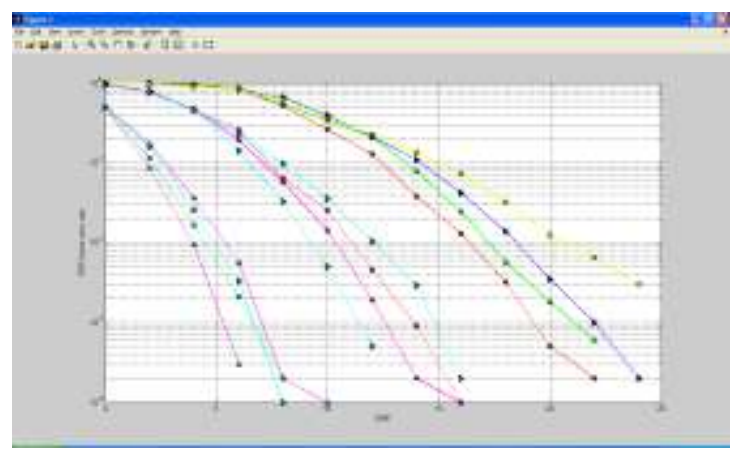

Fig 12 Performance Comparison of the 4-PSK 8-state STTCs from Table 2 (Chen et al.) over correlated Nakagami fading channels $(\mathrm{m}=2)$ for $\rho=0,0.5,0.8$ and 1.0 with

$$
n_{t}=2, n_{r}=1,2 \text { and } 4 \text {. }
$$

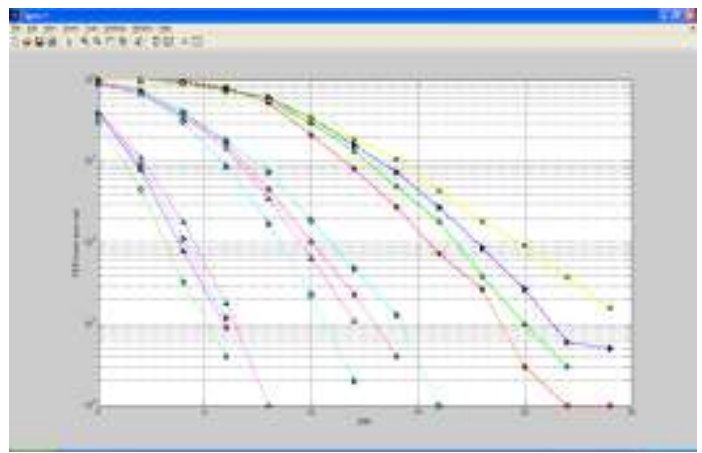

Fig 13 Performance Comparison of the 4-PSK 16-state STTCs from Table 2 (Chen et al.) over correlated Nakagami fading channels $(\mathrm{m}=2)$ for $\rho=0,0.5,0.8$ and 1.0 with

$$
n_{t}=2, n_{r}=1,2 \text { and } 4 \text {. }
$$

\section{CONCLUSION}

We presented the simulation results. From these results we found that the space-time code design criteria proposed for Rayleigh fading channels is suitable for Nakagami fading channels. It was reported in Z. Chen, J. Yuan, B. Vucetic [19] for Rayleigh fading channels with a single receive antenna, that when $n$,is increased from two to three and four, worse performance was degraded for the 4-PSK 4-state STTC in Table 2. In this paper, the performance of STTCs over Nakagami fading channels was presented. We found that the codes designed for Rayleigh fading channels are also suitable for Nakagami fading channels.we observed that in a Nakagami fading channel, this code also perform worse with a single receive antenna, when $n_{t}$ is increased from two to three and from three to four. We found that significant performance improvements can be achieved if we increase the number of transmit antennas from two to three and four. By increasing the number of transmit antennas at the base station a significant performance improvement can be achieved without increasing the burden of the receivers. 


\section{VIII.REFRENCES.}

[1] S. M. Alamouti, "A simple transmit diversity technique for wireless communications," IEEE J. Select. Areas Commun., vol. 16, no. 8, pp. 1451-1458, October 1998.

[2] M. Nakagami, "The m-distribution: A general formula of intensity distribution of rapid fadmg," in Statistical Metho\& in Radio Wave Propagation, W. G. Hoffman, Ed. Oxford, England: Pergarnon, 1960.

[3] Y. Gong and K. B. Letaief, "Performance of space-time trellis coding over Nakagami fading channels" IEEE Vehic. Tech. Co\& pp. 1405-1409, Spring 2001.

[4] G. Bauch, J. Hagenauer, and N. Seshadri, "Turbo processing in transmit antenna diversity systems," Ann. Telecommun., vol. 56, no. 7-8, pp. 455-471, 2001.

[5] V. Tarokh, N. Seshadri, A. R. Calderbank, "Space-time codes for high data rate wireless communication: performance criterion and code construction," IEEE Trans. Innfom. Theoy, vol.44, pp. 744-65, Mar. 1998.

[6] G.L. Stuber, Principles of Mobile Communications. USA, Kluwer Academic Publishers, 2001.

[7] H. Bolcskei and A. J. Paulraj, "Performance of spacetime codes in the presence of spatial fading correlation," in Proc. Asilomar Conference on Signals, Systems and Computers, October 2000, pp. 687-693.

[8] V. Tarokh, A.F. Naguib, N. Seshadri, A.R. Calderbank., "Space-time codes for high data rate wireless communication: performance criteria in the presence of channel estimation errors, mobility, and multiple paths," IEEE Trans. Commtln., vol. 47, pp. 199-207, Feb. 1999.

[9] N. Seshadri, V. Tarokh, A.R. Calderbank," Space-time codes for wireless communication: code construction," IEEE 47th Vehic. Tech.. Conf. Tech.., pp. 637-641, 1997.

[10] S. M. Alamouti, "A simple transmitter diversity scheme for wireless communications," IEEE J. Select. Areas Commn., vol. 16, pp. 1451-1458, Oct. 1998.

[11] M. Damen, A. Abdi, and M. Kaveh, "On the e_ect of correlated fading on several space-time coding and detection schemes," in Proc. IEEE Vehicular Technology Conference, 2001, pp. 13-16.

[12] G. Foschini, "Layered space-time architecture for wireless communication in a fading environment when using multi-element antennas," in Bell Labs Technical Journal, 1996, pp. 41-59.
G. Foschini and M. Gans, "On limits of wireless communication in a fading environment when using multiple antennas," Wireless Personal Communications, vol. 6, pp. 311-335, March 1998.

[14] Y. Gong and K. B. Letaief, "Concatenated space-time block coding with trellis coded modulation in fading channels," IEEE Transactions on Wireless Communications, vol. 1, no. 4, pp. 580-590, Oct 2002.

[15] D. Varshne, C . Arumugam, V. Vijayaraghavan, N. Vijay and S. Srikanth, "Space-time codes in wireless communications," IEEE Potentiah, vol. 22, pp.36 - 38, August-September 2003.

[16] S. Baro, G. Bauch, A. Hansmann, "Improved codes for space-time trellis-coded modulation.," IEEE Commn. Letters, vol.4, no.1, pp.20-22 Jan. 2000.

[17] D. M. Ionescu, "New results on space-time code design criteria" IEEE Wireless Commnnications and Networking Conj, pp. 684-687, 1999.

[18] D. M. Ionescu, K. K. Mukkavilli, Y. Zhiyuan and J. Lilleberg, "Improved 8- and 16-state space-time codes for 4PSK with two transmit antennas," IEEE Commnn. Lett., vol. 5, no. 7, pp. 301-303, July 2001.

[19] Z. Chen, J. Yuan, B. Vucetic, "Improved space-time trellis coded modulation scheme on slow Rayleigh fading channels," Ehctronics Lett., vo1.37, pp.440441,29 Mar. 2001.

[20] Z. Chen, J. Yuan and B. Vucetic," An improved spacetime trellis coded modulation scheme on slow Rayleigh fading channels," IEEE International Conf. Comn., pp. 1110-1116, 2001.

[21] J. Lai and N. B. Mandayam, "Performance of turbo coded WCDMA with downlink space-time block coding in correlated fading channels," accepted for publication in IEEE transaction on wireless communications, 2002.

[22] J. Yuan, 2. Chen and B. Vucetic, " Performance of space-time c o h g on fading channels," IEEE Trans. Commn , vol. 51, no. 12, pp. 1991-1996, Dec. 2003

[23] Y.zhu and H.Zafarkhani "Super pseudo orthogonal space time trellis codes" in proc of IEEE WSNC 2006 vol.3 pp1380-1385 april 2006.

[24] H.Zafarkhani and N.Hassanpour "super quasi orthogonal space time trellis codes for four transmit antenna" IEEE trans on wireless comm.vol.4 pp.215227 jan 2005. 
Table 1. New 8-PSK 2-xtr trellis codes proposed by Chen et al.

\begin{tabular}{|l|c|c|c|c|c|c|c|c|}
\hline states & $\mathrm{a}_{0}{ }^{1}, \mathrm{a}_{0}$ & $\mathrm{a}_{1}{ }^{1}, \mathrm{a}_{1}{ }^{2}$ & $\mathrm{~b}_{0}{ }^{1}, \mathrm{~b}_{0}$ & $\mathrm{~b}_{1}{ }^{1}, \mathrm{~b}_{1}{ }^{1}$ & $\mathrm{~b}_{2}{ }^{1}, \mathrm{~b}_{2}$ & $\mathrm{c}_{0}{ }^{1}, \mathrm{c}_{0}{ }^{2}$ & $\mathrm{c}_{1}{ }^{1}, \mathrm{c}_{1}{ }^{2}$ & $\mathrm{c}_{2}{ }^{1}, \mathrm{c}_{2}{ }^{2}$ \\
\hline 16 & $(2,1)$ & $(3,4)$ & $(4,6)$ & $(2,0)$ & - & $(0,4)$ & $(4,0)$ & - \\
\hline 32 & $(2,4)$ & $(3,7)$ & $(4,0)$ & $(6,6)$ & - & $(7,2)$ & $(0,7)$ & $(4,4)$ \\
\hline
\end{tabular}

Table 2. 4-PSK 3-xtr trellis codes proposed by Chen etc

\begin{tabular}{|c|c|c|c|c|c|c|c|}
\hline states & $\mathrm{a} 0_{0}, \mathrm{a}_{0}{ }^{2}, \mathrm{a} 0$ & $\mathrm{a} 1_{1}^{1}, \mathrm{a} 1^{2}, \mathrm{a} 1^{3}$ & $\mathrm{~b}_{0}{ }^{1}, \mathrm{~b}_{0}{ }^{2}, \mathrm{~b}_{0}{ }^{3}$ & $\mathrm{~b}_{1}{ }^{1}, \mathrm{~b}_{1}{ }^{2}, \mathrm{~b}_{1}^{3}$ & $\mathrm{c}_{0}{ }^{1}, \mathrm{c}^{2}, \mathrm{c}^{2}$ & $\mathrm{c}_{1}{ }^{1}, \mathrm{c}_{1}{ }^{2}, \mathrm{c}_{1}^{3}$ & $\mathrm{c}_{2}{ }^{1}, \mathrm{c}_{2}{ }^{2}, \mathrm{c}_{2}{ }^{3}$ \\
\hline 4 & $(0,2,2)$ & $\begin{array}{l}- \\
-\end{array}$ & $(2,3,3)$ & $\begin{array}{l}- \\
-\end{array}$ & $(1,2,3)$ & $(2,0,2)$ & - \\
\hline 8 & $(2,2,2)$ & $\begin{array}{l}- \\
-\end{array}$ & $(2,0,3)$ & $(1,2,0)$ & $(2,1,1)$ & $(0,2,2)$ & - \\
\hline 16 & $(1,2,1)$ & $(2,2,0)$ & $(2,0,2)$ & $(3,2,1)$ & $(1,3,2)$ & $(2,0,2)$ & $\begin{array}{l}- \\
-\end{array}$ \\
\hline 32 & $(0,2,2)$ & $(1,2,2)$ & $(2,2,0)$ & $(1,2,2)$ & $(2,3,3)$ & $(2,3,1)$ & $(2,0,0)$ \\
\hline
\end{tabular}

Table 3. 4-PSK 4-xtr trellis codes proposed by Chen etc

\begin{tabular}{|c|c|c|c|c|c|c|c|}
\hline States & $\mathrm{a}_{0}{ }_{\mathrm{a} 0}{ }^{2} \mathrm{a}_{0}{ }^{3} \mathrm{a}_{0}^{4}$ & $\mathrm{a}_{1}{ }^{1} \mathrm{a}_{1}{ }^{2} \mathrm{a}_{1}{ }^{\mathrm{a}}{ }_{1}^{4}$ & $\mathrm{~b}_{0}{ }^{1} \mathrm{~b}_{0}{ }^{2} \mathrm{~b}_{0}{ }^{3} \mathrm{~b}_{0}{ }^{4}$ & $\mathrm{~b}_{1}{ }^{1} \mathrm{~b}_{1}{ }^{2} \mathrm{~b}_{1}{ }^{3} \mathrm{~b}_{1}{ }^{4}$ & $\mathrm{c}_{0}{ }^{1} \mathrm{c}_{0}{ }^{2} \mathrm{c}_{0}{ }^{3} \mathrm{c}_{0}^{4}$ & $\mathrm{c}_{1}{ }^{1} \mathrm{c}_{1}{ }^{2} \mathrm{c}_{1}{ }^{3} \mathrm{c}_{1}{ }^{4}$ & $\mathrm{c}_{2}{ }^{1} \mathrm{c}_{2}{ }^{2} \mathrm{c}_{2}{ }^{3} \mathrm{c}_{2}{ }^{3}$ \\
\hline 4 & $(0,2,2,0)$ & -- & $(2,3,3,2)$ & -- & $(1,2,3,2)$ & $(2,0,2,1)$ & -- \\
\hline 8 & $(2,2,2,2)$ & -- & $(2,0,3,1)$ & $(1,2,0,3)$ & $(2,1,1,2)$ & $(0,2,2,1)$ & -- \\
\hline 32 & $(1,2,1,1)$ & $(2,2,0,0)$ & $(2,0,2,2)$ & $(3,2,1,2)$ & $(1,3,2,2)$ & $(2,0,2,2)$ & -- \\
\hline
\end{tabular}

